\title{
EDUCADORES A PENSAR ${ }^{50}$
}

\author{
Educators to think
}

María Natalia del Pilar Duque Higuera*

Duque. M.N. (2016). Educadores a Pensar. Revista PACA, 8, 186-199

\section{Resumen}

Este artículo presenta los resultados de una investigación dirigida a determinar el conocimiento de un grupo representativo de estudiantes (116) de la Facultad de Educación de la Universidad Surcolombiana sobre el tema de abuso sexual infantil (A.S.I.). Estos estudiantes se encontraban en últimos semestres y realizando sus prácticas en instituciones educativas durante el segundo semestre de 2015. Por ser el tema tan amplio, se escudriñó suficiente información en estudios y teorías para determinar los parámetros de la encuesta que se les aplicó on line ${ }^{51}$, diseñada por la propia investigadora. Con los resultados obtenidos se intentó, desde un diseño cuantitativo descriptivo, reflejar e interpretar el conocimiento o desconocimiento del tema de abuso sexual infantil en el área escolar por parte de universitarios que se están preparando para ser docentes. Se tuvieron en cuenta las siguientes variables: el concepto de abuso sexual

\footnotetext{
${ }^{50}$ Síntesis del estudio realizado en 2015 sobre conocimientos de los estudiantes universitarios de últimos semestres (VI-IX) sobre abuso sexual infantil.

"Magíster en Educación. Catedrática del Departamento de Psicopedagogía de la Universidad Surcolombiana. Email: manadelpi@hotmail.com

${ }^{51}$ Encuesta digitada con herramienta Jotform.
} 
infantil, la normatividad que rige actualmente en el Departamento y en el país, la ruta de atención necesaria para atender casos detectados en el aula y las prácticas que ha tenido el estudiante universitario con casos concretos de abuso sexual infantil. El proceso de recolección de datos tardó un tiempo considerable, pero se pudo determinar la apremiante necesidad de capacitar dicha población universitaria sobre abuso sexual infantil. Esto hizo pensar que hay que buscar la conversión de una universidad que sea reconocida por ser dinamizadora de procesos que protegen a los niños y niñas del abuso y el maltrato en la región surcolombiana.

Palabras Clave: abuso sexual infantil, normatividad, ruta de atención y conocimiento.

\section{Summary}

This project was conducted to determine the knowledge of Child Sexual

Abuse (CSA) in education undergraduate students. Participants were 116 senior students of the program and those doing the practices in schools from Surcolombian University in Neiva Huila - Colombia.

The study period covered the second half of 2015. A full scientific study was gathered about Child Sexual Abuse (CSA) supporting scholarly resources. Data was collected through a questionnaire process stating how much knowledge students have in regards to child sex abuse. The questions consisted of self-knowledge variables: The concept of child sexual abuse, the current laws and regulations within the country, and intervention to prevent child sex abuse. Additional questions posed were student's personal experience with child sex abuse in regard to how do they know how to report such cases.

Results from the questionnaire determined the students showed an inadequate level of knowledge, meaning the students cannot process a child sex abuse effectively. The knowledge of Child Sexual Abuse (CSA)'s is unfortunately lower than the international groups.

In conclusion, there is an urgent need of training for these students in 
order for them to become advocates for abused children. The creation of such a program as Child Sexual Abuse (CSA) will help prevent and intervene on behalf of Colombian's future children. This is an ongoing need and will need to be monitored and evaluated as time goes by to insure regulations are being met.

\section{Introducción}

El presente artículo muestra los resultados de una investigación y su propósito fue describir el conocimiento sobre abuso sexual infantil que tenían los estudiantes universitarios de la Facultad de Educación de la Universidad Surcolombiana titulada: "Conocimiento de los estudiantes universitarios de últimos semestres (VI-IX) sobre abuso sexual infantil" durante el segundo semestre de 2015. Para ello se tuvieron en cuenta muchos conceptos de abuso sexual infantil como el de Kempe (Kempe, 1978): "La implicación de niños/as u adolescentes, dependientes e inmaduros, en cuanto a su desarrollo, en actividades sexuales que no comprenden plenamente, y para las cuales son incapaces de dar un consentimiento informado." Este concepto revela un importante aporte a los educadores de niños y niñas, ya que dimensiona la vulnerabilidad y la problemática con la población infantil y la labor de los educadores.

Al entender la dimensión del abuso sexual infantil, se hace indispensable construir conocimiento que permita mostrar el grado de los docentes en cuanto a conceptualizaciones, formas de detección, manejo del abuso sexual infantil y prácticas. Las instituciones educativas no pueden alcahuetear el abuso sexual infantil con el silencio y menos ser incompetentes. Los actores principales, los docentes, deben capacitarse y conocer del tema. No solo deben dirigir los procesos de aprendizaje, siguiendo los distintos modelos pedagógicos, sino que deben vincularse directamente al cuidado integral y a las acciones del buen trato hacia la infancia.

Con esto, la función educadora de la Universidad Surcolombiana va más allá de cumplir con unas normas laborales como preparar clases y diseñar estrategias de enseñanza y aprendizaje. Se convierte también en conocer al estudiantado, vinculándose a su existencia para descubrir sus cualidades individuales y sus falencias; también para observar y analizar la manera como abordarán o ejercitarán sus conocimientos adquiridos en el mundo 
académico para aplicar al mundo real al momento de iniciar sus prácticas universitarias.

El estudio planteado ayudará, entre otros aspectos, a definir el impacto social que posee el conocimiento sobre abuso sexual infantil como una de las funciones de las instituciones de educación superior.

\section{Marco Teórico}

Este estudio pretendió de alguna manera hacer un aporte a los educadores universitarios, quienes deben pensar cómo están desarrollando las prácticas sus estudiantes en las diferentes instituciones educativas y advierten que el tema del abuso sexual infantil es desconocido por ellos. Ignoran qué es el abuso sexual, los criterios para detectarlo, las causas y consecuencias, el manejo para abordar un abuso sexual y las normas existentes.

El educador o docente asesor de práctica universitario, de alguna manera da unas indicaciones que pueden o no dejar desconcertado al practicante, quien debe desarrollar su práctica de todas maneras con niños y niñas ${ }^{52}$ con indicadores de ser abusados. Al final, mejor si no reporta el abuso sexual sufrido por el niño o niña y no se mete líos. Si esos indicadores se hubieran reconocido a tiempo por el practicante llevarían a la detección rápida y eficaz del A.S.I. y un manejo adecuado, pero no estuvo capacitado. Entre los diversos conceptos que teorizan el abuso sexual se encuentran: "Cualquier acción de un familiar que involucre a un menor en actividades de índole sexual. Dentro de este grupo diferenciamos las acciones que incluyen contacto físico de aquellas otras que carecen de este elemento. Dentro de esta tipología se citan el incesto (familiares cercanos), estimulación sexual (tocamientos, masturbación), vejaciones, violación, exhibicionismo, explotación sexual (pornografía infantil, prostitución), etc." (Aguilar, 2009).

\footnotetext{
${ }^{52}$ En términos jurídicos, los menores de edad son aquellos individuos que todavía no alcanzaron la mayoría de edad, como ya dijimos, y por caso están sometidos al régimen que se conoce como patria potestad; esto quiere decir que viven bajo autoridad de sus progenitores que tienen la responsabilidad de protegerlos y de educarlos hasta que cumplan la mayoría de edad. En este artículo se usó este término. Definición tomada de www.definicionabc.com/derecho/menor-de-edad.php
} 
El abuso sexual de un menor es un proceso que consta generalmente de varias etapas o fases; se pueden encontrar en casi todos los autores las mismas etapas con algunas diferencias (Barrios, 2009). La autora Ángela Barrios describe en cada fase unas características que son relacionadas así:

Fase de seducción. El abusador manipula la dependencia y la confianza del menor, y prepara el lugar y el momento del abuso. En esta etapa el abusador conquista al niño, niña o adolescente por medio de regalos o juegos, se acerca paulatina y progresivamente al niño, no necesariamente a nivel sexual, sino encontrándose con él, haciendo actividades juntas (escolares, lúdicas, recreativas, de transporte), favoreciendo progresivamente el contacto, erotizando la relación con expresiones como caricias y besos.

La Fase de interacción sexual abusiva. Es un proceso gradual y progresivo, que puede incluir comportamiento exhibicionista, voyerismo, caricias con intenciones eróticas, masturbación. En este momento ya se puede hablar de "abusos sexuales" (Barrios, 2009). La fase donde se da el coito propiamente dicho se produce en un momento bastante avanzado de la interacción sexual abusiva.

La Instauración del secreto. Generalmente por medio de amenazas impone el silencio en el menor, a quien no le queda más remedio que adaptarse. El agresor busca mantener la circunstancia abusiva en secreto a toda costa, argumentando al niño que lo que ha pasado es un secreto de dos, que ha ocurrido porque se quieren (Barrios, 2009).

La Fase de divulgación. Si llega puede ser accidental o premeditada, el menor decide contar lo que está pasando porque ya no lo tolera. Puede ocurrir por accidente, al ser sorprendidos por un tercero que advierte que debe denunciar el hecho.

La Fase represiva. Se presenta después de la divulgación, en el caso del incesto la familia busca desesperadamente un reequilibrio para mantener a cualquier precio la cohesión familiar, por lo que tiende a negar, a restarle importancia, a justificar el abuso, o a presionar al niño o niña para que se retracte de lo expresado en la fase de divulgación, en un intento por seguir como si nada hubiese sucedido. 
Las tipologías del A.S.I. (REDIME, 2010) son de dos formas: con contacto físico cuando hay penetración vaginal, anal u oral, penetración digital o con objetos. Este contempla los tocamientos, caricias, toques y manoseos. Y sin contacto físico, de manera verbal cuando hay palabras eróticas, proposiciones indecentes y proposiciones verbales explícitas. Abuso visual, con exposición del cuerpo, masturbación y pornografía. Psicológico, si sobrepasan los límites emocionales apropiados entre adultos y niños, padres e hijos (por ejemplo contar intimidades de la pareja, convertir a la hija en "esposa o al hijo en el "marido" emocionalmente).

Todo profesor debe saber sobre las consecuencias psicológicas. Teniendo en cuenta a Rodríguez y colaboradores (Rodríguez, 2012) se dice que los efectos a largo plazo son, comparativamente, menos frecuentes que las consecuencias iniciales; sin embargo, el abuso sexual infantil constituye un importante factor de riesgo para el desarrollo de una gran diversidad de trastornos psicopatológicos en la edad adulta.

Algunos autores, entre ellos Peleikis, constatan una peor salud mental general en víctimas de abuso sexual infantil, con una mayor presencia de síntomas y trastornos psiquiátricos (Peleikis, 2005).

Los niños son grabadoras encendidas 24 horas al día. La formación de la identidad se da en los primeros años de la vida, por eso todo daño causado en esa etapa, tiene consecuencias muy serias. Por ejemplo, los efectos cognoscitivos propuestos por Portero (Portero, 2011) señalan que se relacionan con el cambio en la autopercepción. Según el autor, el niño o niña desarrolla una sensación interna de "maldad" como una forma de justificar el abuso y al agresor. Estos cambios producen daño cognitivo a nivel del juicio crítico, lo que impediría la evaluación de situaciones de riesgo y la consecuente vulnerabilidad a la victimización en la etapa adulta. Respecto a las normas, desde hace muchas décadas estas se han venido fortaleciendo, reestructurando los derechos y sumando esfuerzos para proteger la infancia ante las distintas formas de violencia. En el marco del abuso sexual en nuestro contexto colombiano encontramos bastante literatura al respecto, recordando que los derechos de los niños y las niñas prevalecen sobre los derechos de los demás (Barrios, 2009). Entre ellos tenemos: la Ley 1146 de 2007 (Julio 10), por medio de la cual se expiden normas para la prevención de la violencia sexual y atención integral de los 
niños, niñas y adolescentes abusados sexualmente. Y la Ley 1098 de 2006 (Noviembre 8), "Por la cual se expide el Código de la Infancia y la Adolescencia". A nivel internacional tenemos la Declaración de los Derechos del Niño y la Convención sobre los Derechos del Niño, adoptada por la Asamblea General de las Naciones Unidas el 20 de noviembre de 1989; en Colombia entró en vigor el 28 de enero de 1991, mediante la Ley 12 de enero 22 de 1991.

La Ruta de Atención para Casos de Abuso Sexual en el Municipio de Neiva (ICBF 2016) va dirigida a unir los esfuerzos de los diferentes organismos que protegen y atienden casos de abuso sexual contra la infancia. La Ruta de Asistencia para Violencia Intrafamiliar y Abuso Sexual, de la Secretaria de Educación y el ICBF de Neiva de 2010, remite el caso a Fiscalía y al ICBF. Luego pasa a manos del Defensor de Familia para la garantía y restablecimiento de los derechos de los niños, niñas y adolescentes que han sido vulnerados. A cada caso se le da asistencia jurídica, psicológica y alojamiento temporal con diferentes organizaciones pertenecientes a la red de apoyo.

Las instituciones que reciben la denuncia o noticia criminal son: Unidad de Delitos Sexuales, Fiscalía, URI-SAU, Sijín, Dijín, Comisarías de Familia, estaciones de Policía, Casa de Justicia, inspecciones de Policía, personal con funciones de Policía Judicial, línea de atención telefónica 112, Instituciones que pueden dictar medidas de protección al niño o niña. El ICBF interviene a través de sus defensores en los centros zonales, Comisarías de Familia y Fiscalía.

\section{Metodología}

El enfoque del estudio fue cuantitativo descriptivo transversal. Según la naturaleza del problema, el trabajo de investigación corresponde a un diseño no experimental, ya que no intenta probar de manera experimental las relaciones causales entre variables.

Durante el proceso de cuantificación numérica, el instrumento de medición o de recolección de datos usado y preferido por la investigadora fue la encuesta on line, validada por 3 expertos quienes determinaron que era confiable y media los objetivos propuestos. 
Los datos recogidos y sistematizados por el programa Excel sirvieron para describir, graficar y cruzar la información entre diferentes variables propuestas. El diseño de estudio es descriptivo transversal (una sola cohorte) porque busca especificar características o perfil de un grupo que se sometió a análisis y donde no existe continuidad en el eje tiempo, porque la medición se dio solo en un momento único.

La población estudiantil perteneció a la Facultad de Educación de la USCO, de los semestres de VI a IX durante el segundo semestre del 2015. La técnica de muestreo para seleccionar los estudiantes fue la de muestreo aleatorio simple porque se numeraron los elementos de la población y se seleccionaron al azar $\mathrm{n}$ elementos que contiene la muestra número representativo de la población. El tamaño de la muestra se calculó con la fórmula:

$$
n=\frac{N \sigma^{2} Z^{2}}{(N-1) e^{2}+\sigma^{2} Z^{2}}
$$

Es así como la población estuvo constituida por 1750 estudiantes de la Facultad de Educación y el tamaño de la muestra de 116 estudiantes universitarios de la Facultad de Educación de los distintos programas. Para los criterios de inclusión estuvieron participando voluntariamente estudiantes universitarios de pregrado que cursaban de VI a IX semestre en los diferentes programas de la Facultad de Educación. Participaron ambos géneros, hombres y mujeres, no importó su edad ni su condición socioeconómica, ni que tuvieran o no un conocimiento sobre el tema de abuso sexual infantil. Sí fue esencial que estuvieran matriculados o activos en los programas o licenciaturas y realizando prácticas en centros escolares públicos.

Se tuvieron en cuenta las siguientes variables: el concepto de abuso sexual infantil, la normatividad que rige actualmente en el Departamento y en el país, la ruta de atención necesaria para ocuparse de los casos detectados en el aula y las prácticas que ha tenido el estudiante con casos concretos de abuso sexual infantil. 


\section{Técnicas e Instrumentos}

El instrumento que se utilizó en el estudio fue la encuesta forma On line. La encuesta incluyó algunas variables sociodemográficas (sexo, programa académico, edad y lugar de origen) y se compone de dos partes diferenciadas. La primera parte consta de preguntas que recogen el conocimiento del estudiante sobre el abuso sexual infantil (Por ejemplo: capacitaciones ha recibido sobre abuso sexual?, Responda falso o verdadero según piense: ¿las personas que abusan de niños o niñas presentan graves patologías mentales o perversiones mentales?). Una última pregunta hace referencia a la experiencia directa del estudiante con estos casos (Por ejemplo ¿Conoces a alguien que haya sufrido abuso sexual en su infancia?'). Estas cuestiones presentan un formato de respuesta dicotómica verdadero/ falso, selección múltiple con única respuesta, selección múltiple con múltiple respuesta. Otros ítems se dirigen a analizar en el encuestado de algunas prácticas y conocimientos de normatividad (Por ejemplo ¿Se han notificado casos de abuso en su lugar de práctica? ¿La institución ha hecho seguimiento del caso?, ¿Conoce alguna norma que regule el ASI?). Lo respondieron de forma dicotómica (verdadero/ falso).

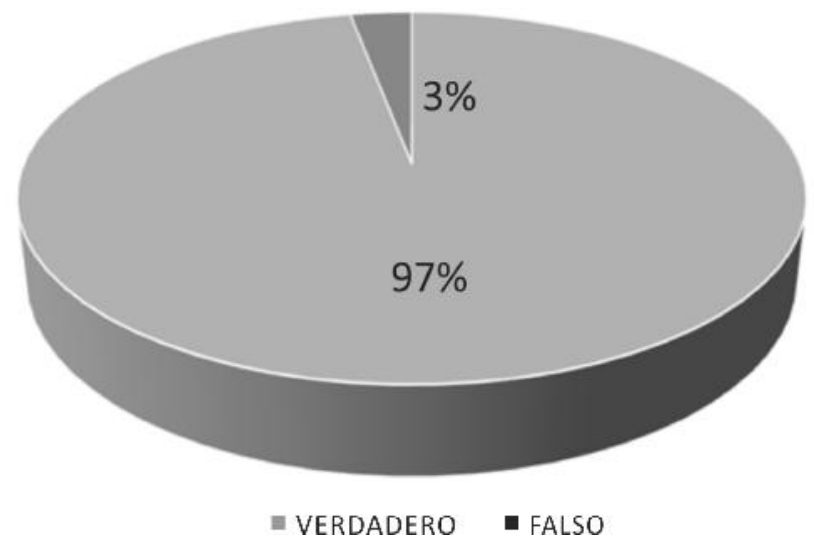

Gráfica 1: ¿Quién realiza el abuso es un desconocido?

Esta gráfica No.1 revela por ejemplo que el 97,41 \% de los universitarios encuestados refirieron que es falso que el A.S.I sea realizado siempre por un desconocido y 2, 59 \% que es verdad esta última proposición. 
Esta respuesta deja ver la incomprensión de los estudiantes analizados. La doctora Irene Intebi, experta en abuso sexual infantil, explica que son los padres biológicos los principales responsables de los abusos intrafamiliares y refiere el estudio llevado a cabo en Buenos Aires entre 1989 y 1992 sobre 138 casos. Los datos son incuestionables: el 42,5\% de los abusadores son los padres biológicos. En segundo lugar, aparecen los familiares cercanos -incluyendo tíos, abuelos, hermanos, primos, etcéteraque representan el $23,7 \%$. El tercer lugar corresponde a los conocidos no familiares, con el 17,5\% " (Intebi, 1998).

También la investigación arroja datos para poder decir sin temor que no existe un currículo, o curso en pregrado que potencialice estos conocimientos. Generalmente los estudiantes dicen: "En la $U$ no me enseñaron nada o lo que sé es porque un día asistí a un curso donde hablaron de eso". iClaro! No solo encontrarán problemas de aprendizaje en niños y niñas sobre matemáticas, español, ciencias, arte, etc., sino que tendrán niños, niñas y adolescentes "difíciles", "apáticos", "llorones" o "extraños", con indicios de abuso sexual.

El punto de quiebre de esta situación puede verse desde varios enfoques a la vez, entre ellos que desde la función docente no se toman en cuenta esos "otros" conocimientos que permiten la formación integral del universitario surcolombiano. Sus aprendizajes no deben ser solo los del currículo del programa, sino de otros temas que complementan su formación. Es comprensible que en las aulas de clase de las diferentes I.E. donde desempeñan las prácticas se evidencian casos donde los infantes y adolescentes sufren de abuso sexual pero que no saben cómo tratar ese caso difícil, porque no están entrenados y capacitados ni mental ni psicológicamente; sin embargo, se convierten en testigos de este flagelo, a veces sin poder decir o hacer algo. ¿Quién se va a imaginar la posibilidad de encontrar esos casos difíciles y quién puede decir exactamente qué hacer?

La expectativa con los educadores es que profundicen en unos saberes más elevados, más significativos y específicos, como por ejemplo, las fases en que ocurre el abuso sexual. Solo un $7,8 \%$ contesta acertadamente las fases del A.S.I. Limitante del conocimiento muy preocupante porque si repasamos lo que ocurre en cada una de ellas, el trabajo de detección en 
el aula debe ser preciso, casi tener ojo clínico y con los cinco sentidos puestos. (Ver anexo No. 1).

Respecto a la Ruta de Atención, en caso de detectar un A.S.I. en la institución educativa, más del 90,52\% no sabe qué es la ruta de atención (Ver anexo No.2). Es decir no conocen qué pueden hacer, cómo direccionar el caso o a qué institución; para un niño esto es un sufrimiento.

Así mismo, la investigación lleva a descifrar con respecto a si conocen sobre las patologías que puede tener un abusador, el 91,38\% respondió que es cierto que todas las personas que abusan de niños o niñas presentan graves patologías mentales o perversiones sexuales. Los abusadores sexuales son heterogéneos en las características de personalidad y psicopatología. Según González, los abusadores "pueden o no tener trastorno de personalidad...del tipo limítrofe, con dificultad en el control de impulsos y en lograr relaciones de intimidad. En el caso de violadores es más frecuente el trastorno de personalidad antisocial, refiere el mismo autor. En general, presentan distorsiones cognitivas, dificultades en el desarrollo de empatía y en la habilidad de entender y atribuir estados mentales a otros, lo que en la literatura se ha denominado la teoría de la mente. Tienen especial habilidad para identificar niños vulnerables (González, E., 2004).

Los estudiantes también presentan una grave falencia al responder que un menor si presenta infección venérea en áreas genitales o boca es por falta de higiene y no por que ha sido abusado sexualmente (46\%). ¿Por qué piensan eso? ¿Acaso es normal que un niño o niña tenga infecciones en sus genitales o boca? Es a los profesores a quién el niño o niña le cuenta que está siendo abusado sexualmente, o a quien informa que le duelen o le rascan los genitales. Pero el desconocimiento del ASI por parte de los educadores conduce a no prestar atención a lo que el niño o niña le está diciendo. Los docentes deben saber que cuando se detecta un abuso sexual casi siempre hay evidencia segura de al menos una Infección de Trasmisión Sexual (I.T.S) como Neiseeria Gonorrhoeae y la sífilis.

Otro mito de los universitarios es que respondan que los niños y niñas pueden evitar la mayoría de las veces el A.S.I con un 65,52\% (Ver anexo 
No.3); esto es una limitante en la tarea de la prevención del abuso sexual. Estos mitos, estereotipos o falsas creencias pueden conceptualizarse como prejuicios, actitudes y creencias, generalmente falsos pero amplia y persistentemente mantenidos, que sirven para negar y justificar las agresiones sexuales que se cometen contra la infancia (Lonsway, 1993). Con esto es posible pensar que los mitos se acomodaron en los universitarios encuestados para justificar que los niños y niñas "tienen lo que se merecen y se merecen lo que tienen"; por tanto, las acciones violentas como la agresión sexual son justas.

El panorama de la investigación manifiesta claramente que un docente o practicante, por no estar preparado y entrenado, puede concebir un ASI como algo natural, normalizar el abuso sexual. Esta normalización del ASI no es lo normal, y el conceptuarlo como normal y no brindar protección al niño o niña no lo merecen en ninguna etapa de su vida, ni en ningún lugar del mundo.

Por ejemplo, el profesor o profesora a cargo del aula o practicante, debe observar cómo es la interacción del adulto cuidador (el que lo lleva y recoge de la I.E.), si recibe constantes regalos de él o ella o sí cuenta que alguien juega con él o ella, que pregunte a qué juegan y en dónde y a qué horas. Si tiene secretos, cuáles son. Son señales de alerta del A.S.I., las cuales van desde cambios físicos, de comportamiento, de hostilidad, de desobediencia, de ánimo, de baja autoestima, de aislamiento, de culpa, de llanto, de miedo, de un repentino bajón en el rendimiento escolar, de hipersexualidad o de juegos sexuales.

Paralelo a esto y como refiere Barrios (2009), un abusador comienza su "macabro juego" con gestos de exhibicionismo, paseándose semidesnudo delante de su víctima, o dejando al descubierto sus órganos sexuales hasta que ocurre lo peor. Cuando el niño o niña le cuenta al profesor sobre el ASI sufrido, el docente debe saber qué hacer. De lo contrario, el abusador intenta mantener el secreto amenazando, chantajeando y culpabilizando al mismo niño, para perpetuar el abuso sexual.

"Una parte del descreimiento hacia las descripciones del abuso que realizan los niños emana del desconocimiento que, en general, se tiene acerca de la capacidad de registro y de almacenamiento de recuerdos en la infancia, 
de cómo impacta una situación traumática sobre un sujeto en desarrollo y de cuáles son los recursos con los que cuenta para comunicar lo que está sucediendo" (Intebi, 2011).

Respecto a esto, Kelley (2008) da unas pautas para detectar bajo rendimiento ligado a A.S.I: dificultades de concentración, desinterés por las actividades vinculadas al aprendizaje, aislamiento del grupo de pares, negativa repentina a participar de actividades físicas, de evitar el contacto físico con cualquier persona, el descenso brusco del rendimiento escolar, dificultades en la capacidad de memoria y atención. Estos se convierten en indicadores en el aula de que algo está ocurriendo con los niños y niñas.

De ahí la necesidad de que los profesores conozcan estas situaciones para descubrir a tiempo si un niño está punto de ser abusado o si ya está siendo abusado.

\section{Conclusiones}

Los estudiantes universitarios no conocen sobre Abuso sexual Infantil en conceptos generales porque no han recibido en la universidad un curso o capacitación sobre el tema. Tienen muchos mitos sobre que es el abuso sexual y no pueden conceptualizarlo, ni especificar fases, tipologías, causas, consecuencias y factores de riesgo.

Es claro que los estudiantes no conocen qué es una ruta de atención, su importancia, su utilidad y no conocen como funciona para el Municipio de Neiva.

Esta investigación confirma el desconocimiento de las normas establecidas sobre el abuso sexual infantil en Colombia, que dirigen su atención al restablecimiento de los derechos de los niños y niñas, el cuidado y la protección.

Las prácticas que han tenido en casos de abuso sexual ha sido poca o nula, bien sea porque no conocen de casos concretos en su lugar de práctica o bien la institución educativa no los ha detectado o no les ha informado. 


\section{Referentes Bibliográficos}

- Aguilar, M. (2009). ABUSO SEXUAL EN LA INFANCIA. Revistas Científicas de la Universidad de Murcia. Anales de Derecho, 210 -240.

- Alokan, F. B. (Abril de 2014). European Scientific Journal . Recuperado el Dox mil diez y xeix

- Arendt, H. (2002). La vida del espíritu. Barcelona: Paidós.

- Arendt, H. (2005). La condición humana. Barcelona: Paidós.

- Barrios, A. (10 de Octubre de 2009). Diseño de una cartilla como herramienta para la prevencion del abuso sexual infantil en niños de 6 a 9 años. Recuperado el 2016, de http://www.bdigital.unal.edu.co/8484/ 1/534793.2009.pdf

- Bunge,M. (2007).

- FISCAL, M. P. (9 de Noviembre de 2015). (ATAJO, Editor) Recuperado el Febrero de 2016, de https://www.fiscales.gob.ar/atajo/capacitacion-adocentes-sobre-abuso-sexual-en-ninos-ninas-y-adolescentes/ ICBF ALCALDÍA DE NEIVA, S. D. ( 2016). Colombia aprende. Obtenido de http://www.colombiaaprende.edu.co/html/familia/1597/articles305952_neiva.pdf

- Intebi, I. (1998). Abuso sexual infantil en las mejores familias, "Perfil del abusador, ¿quiénes son? Madrid. España.

- Kelley, S. J. (2008). El Abuso Sexual de Niños en Guarderías. . ASAPMI Asociacion Argentina de Prevención del Maltrato Infantil y Juvenil.

- Kempe, H. (1978). Sexual abuse, another hidden pediatric problem. SPediatrics, 62: 182-8.

- Lonsway, K. (1993). Rape Myths. sage journal, 133-164.

- Lopez, F. (1996). Los abusos sexuales de menores. lo que recuerdan los adultos. Anales de psicología, 196.

- Peleikis. (2005). Currentt mental health in women with childhood sexual.

- Portero, G. (1 de diciembre de 2011). Euskadi. (E. J. Nagusia, Ed.) Recuperado el 15 de octubre de 2016.

- REDIME. (19 de Junio de 2010). Obtenido de Asociación Redime Blog: http://redimeasociacion.blogspot.com.co/2010/06/diferentes-tipos-deabusos-sexuales_1795.html

- Rodríguez, Y. (2012). Consecuencias Psicológicas del Abuso Sexual Infantil. Periódicos Eletrónicos de Psicologia (PePSIC), 9(1). 